\title{
SUB-SAMPLE INTERPOLATION STRATEGIES FOR SENSORLESS FREEHAND 3D ULTRASOUND
}

R. J. Housden, A. H. Gee, G. M. Treece and R. W. Prager

\section{CUED/F-INFENG/TR 545}

January 2006

University of Cambridge

Department of Engineering

Trumpington Street

Cambridge CB2 1PZ

United Kingdom

Email: rjh80/ahg/gmt11/rwp @eng.cam.ac.uk 


\title{
Sub-sample interpolation strategies for sensorless freehand 3D ultrasound
}

\author{
R. James Housden, Andrew H. Gee, Graham M. Treece and Richard W. Prager \\ University of Cambridge \\ Department of Engineering \\ Trumpington Street \\ Cambridge CB2 1PZ
}

\begin{abstract}
Freehand 3D ultrasound can be acquired without a position sensor by deducing the elevational probe motion from the inter-frame speckle decorrelation. However, a freehand scan involves lateral and axial, as well as elevational, probe motion. The lateral sampling is determined by the A-line separation and is relatively sparse: lateral motion tracking therefore requires sub-sample interpolation. In this paper, we investigate the resilience of lateral interpolation techniques to simultaneous lateral and elevational probe motion. We propose a novel interpolation strategy and, through a series of in vitro experiments, compare its performance with that of established alternatives. The new technique is shown to be superior, limiting interpolation errors to around $5 \%$ of the length of the freehand reconstruction.
\end{abstract}

\section{Introduction}

Freehand 3D ultrasound can be acquired without a position sensor by exploiting speckle decorrelation $[4,12,16]$ to determine out-of-plane transducer motion. Consider two adjacent B-scans $A$ and $B$ in a freehand sequence, as shown in Figure 1. Since the focusing of the ultrasound beam is far from perfect, the backscattered signal at any point is a function of the scatterers in a certain resolution cell around that point. The resolution cells are elongated in the elevational direction and there is considerable overlap between cells in neighbouring B-scans. It follows that if we examine corresponding patches on $A$ and $B$, we will observe a correlation between the two sets of backscattered signals. Moreover, the correlation depends on the degree of overlap of the resolution cells, and hence the separation of the two patches. We can therefore measure the interpatch correlation and look up the corresponding separation on a decorrelation curve, as illustrated in Figure 1. A minimum of three such lookups, for three (non-collinear) pairs of patches, allows us to estimate the elevational separation, tilt and yaw of $A$ relative to $B$. Any in-plane motion between $A$ and $B$ (translation in the axial and lateral directions, roll around the elevational axis) can be determined using $2 \mathrm{D}$ image registration techniques [14, 15].

The precise form of the elevational decorrelation curve can be derived from ultrasound physics or, more practically, by scanning a speckle phantom and measuring $\rho$ for different elevational offsets $d_{e}$. Since the focusing varies across the image, particularly with depth, we require a different decorrelation curve for each location in the B-scan.

There are several difficulties with this approach that impede its direct application to in vivo freehand data. First, the theory holds only for fully developed speckle. Real tissue contains regions of coherent scattering which decorrelate at a slower rate than regions of fully developed speckle. In recent work [8], we have shown how to allow for this in an adaptive speckle decorrelation framework. Secondly, there are other causes of speckle decorrelation (noise, transducer rotation, tissue compression, physiological motion) which might be confused for elevational motion. This is an open problem and a matter for future research. Finally, there is the need for sub-sample interpolation, which is the subject of this paper.

Consider the illustration in Figure 2, which shows regions of two B-scans $A$ and $B$ of a spherical object. Between $A$ and $B$ there is out-of-plane, elevational motion - which causes the circle in $B$ to appear smaller than the circle in $A$ - and in-plane, lateral motion - which causes the circle to shift to the right from $A$ to $B$. To determine the position of $B$ relative to $A$, we must first 


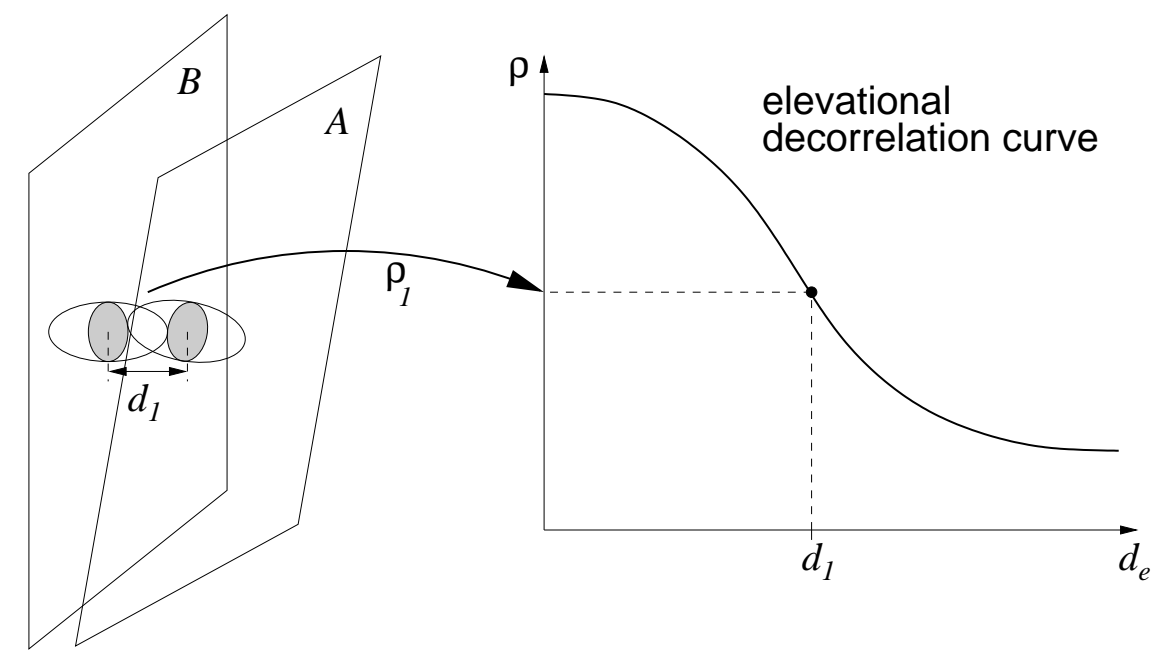

Figure 1: Principle of elevational speckle decorrelation. Consider corresponding patches in scans $A$ and $B$ (the shaded ellipses). Because of the imperfect elevational focusing, the contents of the patches depend on scatterers within overlapping resolution cells (the hollow ellipsoids) and are therefore correlated. The correlation coefficient depends on the degree of overlap and hence the elevational separation. It follows that, given a suitable decorrelation curve, a measured correlation $\rho_{1}$ can be used to look up the corresponding separation $d_{1}$.

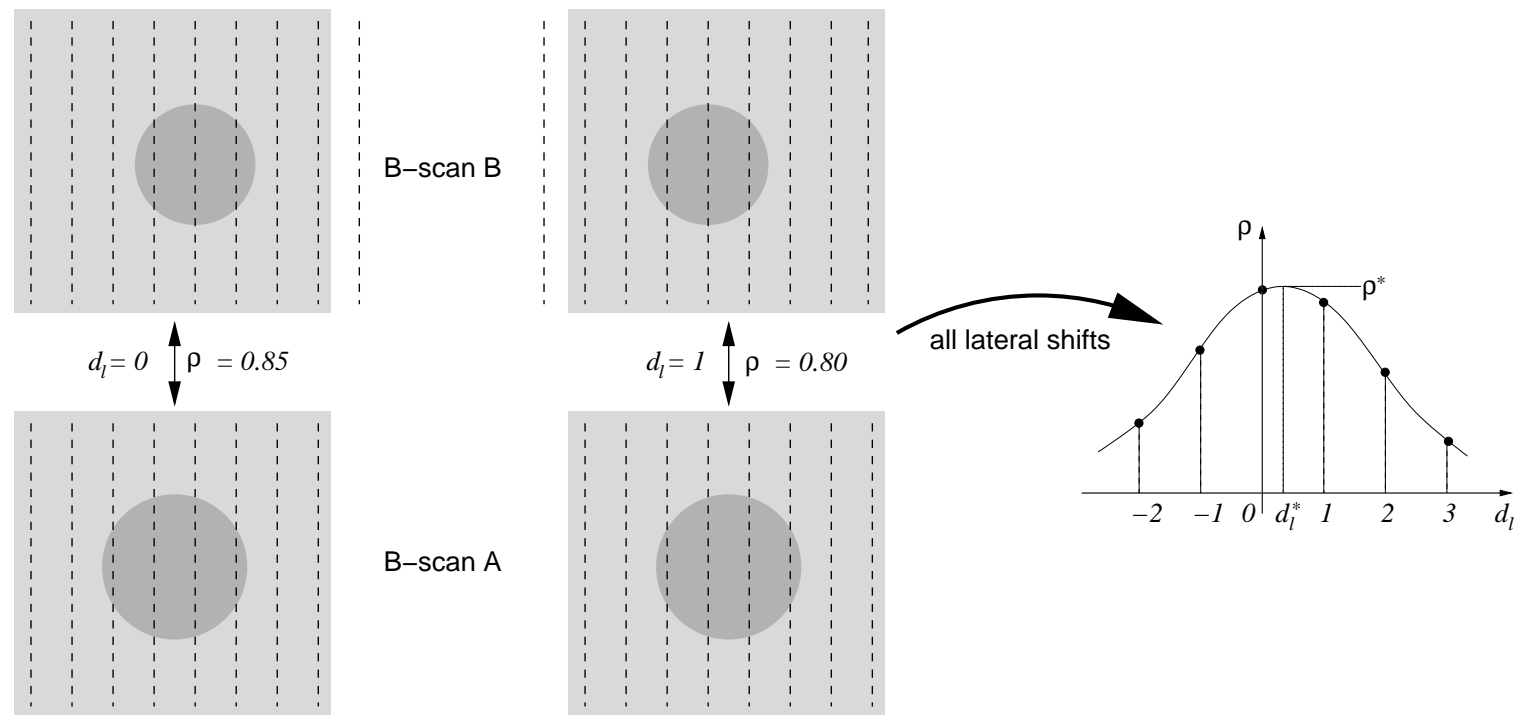

Figure 2: Sub-sample interpolation in the lateral direction. Each dashed line represents an A-line (vector) of radio frequency echo data. Before a patch on B-scan $A$ can be correlated with a patch on B-scan $B$, to determine the elevational offset, it is first necessary to find the corresponding patch on $B$, allowing for any lateral and axial motion between $A$ and $B$. The patch on $A$ is therefore correlated with a number of candidate patches on $B$ within a suitable search window: the highest correlation indicates the correct lateral and axial shifts. The diagram illustrates two candidate matches, one with no lateral shift $\left(d_{l}=0\right.$, left $)$ and one with a single vector shift $\left(d_{l}=1\right.$, right). Since the lateral sampling is relatively sparse, sub-vector interpolation is necessary to determine the correct shift $d_{l}^{*}$ and the peak correlation value $\rho^{*}$. 
determine the correct lateral shift and then the correlation at this shift (the correlation is used to determine the elevational offset, as described above). The problem is that the lateral sampling is sparse, with one A-line per transducer crystal. Correlating the $A$ patch with $B$, with no lateral shift, might give a correlation coefficient of, say, 0.85. Correlating one vector to the right gives, say, 0.8. The correct lateral shift is somewhere in between, at a shift of $d_{l}^{*}$ with a peak correlation $\rho^{*}$. But how do we determine $\rho^{*}$ and $d_{l}^{*}$ ?

It is important to note that sub-vector interpolation is essential: rounding to the nearest whole vector would seriously compromise any $3 \mathrm{D}$ reconstruction. For high frame rate acquisition, the lateral shift between adjacent B-scans is often less than 0.5 vectors: rounding down to zero would result in 3D reconstructions exhibiting little lateral probe motion, even though the actual motion might be considerable when accumulated over several hundred B-scans. Similarly, using the highest measured $\rho$ instead of the interpolated $\rho^{*}$ would also compromise reconstruction accuracy. Since $\rho \leq \rho^{*}$, correlations would be consistently underestimated and elevational offsets consequently overestimated.

We should also note that interpolation is an issue in the lateral direction only, since this is where the sampling is sparse. For linear array probes, typical crystal pitches are around one Aline per $0.3 \mathrm{~mm}$ in the lateral direction. In contrast, axial definition is far superior, with perhaps $0.01 \mathrm{~mm}$ per sample for typical digitisation of the radio frequency $(\mathrm{RF})$ echo signal. Rounding to the nearest sample in the axial direction is highly unlikely to compromise $3 \mathrm{D}$ reconstructions of long B-scan sequences.

The most important quality we are seeking in a lateral interpolation algorithm is that it should be unbiased. In our sensorless freehand 3D ultrasound application, lateral and elevational motion are accumulated over many B-scans, but interpolation errors will not grow as long as there is no bias. Conversely, any bias will cause drift errors in long sequence reconstructions.

Sub-sample interpolation has been studied previously for two main applications: velocity estimation by speckle tracking (as an alternative to conventional Doppler imaging) and strain estimation for elastography. The latter helps explain why much of the previous work concerns interpolation in the axial direction. One of the few papers to address lateral interpolation is [9], in which the grid slopes algorithm is shown to be relatively unbiased in comparison with other techniques. More prone to bias are cosine [3, 5, 6], cubic spline [9] and parabolic interpolation $[2,3,7]$. Fourier reconstruction [3] has been shown to work well in the axial direction, provided the signal is bandlimited and the sampling frequency above the Nyquist limit.

There are two major differences between this body of previous work and the study presented in this paper. First, for elevational motion estimation we are interested not only in the lateral shift $d_{l}^{*}$ but also the correlation peak $\rho^{*}$. Secondly, our data exhibits deliberate and significant elevational motion which may compromise the interpolation techniques' ability to determine $d_{l}^{*}$. We are not aware of any previous work that has addressed these points. The literature on sensorless freehand 3D ultrasound has largely ignored the interpolation issue.

This paper is organised as follows. In Section 2, we describe the various interpolation strategies under consideration. Section 3 contains details of the experimental methodology employed to evaluate the techniques, along with the results. Finally, in Section 4 we draw some conclusions and suggest avenues for further work.

\section{Interpolation strategies}

\section{Interpolating the data}

Ultrasound machines typically display B-scans with more lateral pixels than there are A-lines. This is achieved by upsampling in the lateral direction, interpolating extra values between adjacent Alines. We consider here two interpolation techniques, one at each end of the speed-accuracy trade-off. Linear interpolation is relatively quick but, of course, fails to capture any curvature in the underlying function. In contrast, a full Fourier reconstruction is slow but, assuming the underlying function is band-limited and sampled above the Nyquist limit, gives perfect results. If 


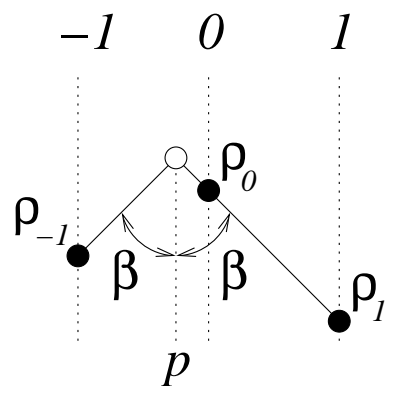

$$
p=\left\{\begin{array}{lll}
\frac{1}{2} \frac{\rho_{1}-\rho_{-1}}{\rho_{0}-\rho_{1}} & \text { if } & \rho_{-1}>\rho_{1} \\
\frac{1}{2} \frac{\rho_{1}-\rho_{-1}}{\rho_{0}-\rho_{-1}} & \text { if } & \rho_{-1}<\rho_{1} \\
0 & \text { if } & \rho_{-1}=\rho_{1}
\end{array}\right.
$$

Figure 3: Triangular interpolation. Given the peak correlation sample $\rho_{0}$ and its two neighbours $\rho_{-1}$ and $\rho_{1}$, the peak location $d_{l}^{*}$ is offset by $p$ from the location of $\rho_{0}$. Given $p$, it is straightforward to derive the corresponding peak correlation $\rho^{*}$.

the ultrasound signal at lateral position $x$ is $a(x)$, and we have samples of $a(x)$ at $x=n \Delta$, where $\Delta$ is the distance between A-lines, then Fourier reconstruction proceeds according to

$$
a(x)=\sum_{n} a(n \Delta) \operatorname{sinc} \frac{\pi(x-n \Delta)}{\Delta},
$$

where $\operatorname{sinc}(x) \equiv(\sin x) / x$.

Care must be taken to minimise the computational expense of using data interpolation to find $d_{l}^{*}$. With reference to Figure 2, we interpolate only B-scan $B$ between the original A-lines, leaving B-scan $A$ as it is. We can then correlate the patch in B-scan $A$ with shifted patches in B-scan $B$, but now with non-integer shifts. This may still be prohibitively slow, since we need to correlate at every upsampled shift position to find the peak correlation to sub-vector precision. Data interpolation may nevertheless play a role in determining $\rho^{*}$ : we could find $d_{l}^{*}$ by some other means, resample the patch in B-scan $B$ at this unique shift, then correlate once to find $\rho^{*}$.

\section{Interpolating the correlation}

Instead of interpolating the data, we could interpolate the correlation $\rho$ between the measured samples. Referring to Figure 2, this involves fitting some function $f\left(d_{l}\right)$ to the available samples of $\rho$, then determining $d_{l}^{*}$ and $\rho^{*}$ from the peak of $f\left(d_{l}\right)$.

The most natural choice for $f\left(d_{l}\right)$ is the true shape of a lateral decorrelation curve, which is approximately Gaussian when imaging fully developed speckle at the beam's focus and correlating the intensity or amplitude envelope of the $\mathrm{RF}$ echo signal $[14,17,18]$. Away from the focus, there are side-lobes in the point spread function and a Gaussian curve is no longer appropriate. As discussed in Section 1, researchers have investigated many different functions $f\left(d_{l}\right)$ for this purpose. Here, we once again consider two functions at opposite ends of the speed-accuracy spectrum. The fastest way to estimate the correlation peak is to fit a symmetric triangular function to the highest three samples [15], as illustrated in Figure 3. This produces results which are very similar to quadratic interpolation through the three highest samples, but is slightly quicker to compute. Far slower, though insignificant compared with the expense of obtaining the samples in the first place, is to fit a Gaussian function to the samples. There is no closed form solution to the fitting problem: instead, we use an iterative optimisation algorithm (Levenberg-Marquardt [13]) to find the best least squares fit. Bearing in mind that the peak correlation will generally be less than one, since there is elevational motion, we consider a three-parameter, scaled Gaussian of the form

$$
f\left(d_{l}\right)=A e^{-\frac{\left(d_{l}-c\right)^{2}}{2 \sigma^{2}}}
$$

which we fit to the peak sample and its two immediate neighbours. We also consider a four- 
parameter, offset scaled Gaussian of the form

$$
f\left(d_{l}\right)=A e^{-\frac{\left(d_{l}-c\right)^{2}}{2 \sigma^{2}}}+B
$$

Since this does not decay to zero, it captures, to some extent, the shape of the lateral decorrelation curve away from the beam's focus. We fit the offset Gaussian to the peak sample and its four immediate neighbours, two to the left and two to the right. We are not aware of any previous work describing Gaussian interpolation between A-lines. In [1], the author describes fitting a theoretical correlation curve, but gives no details of the nature of the curve or how it is fitted.

For comparison, we also consider the published state-of-the-art for lateral interpolation, the grid slopes algorithm. We do not describe the algorithm here, but rather refer the reader to [9]. However, we must point out that the algorithm is specifically formulated for the sum of absolute differences similarity measure, and not the correlation coefficient, so we can only use grid slopes to determine $d_{l}^{*}$ and not $\rho^{*}$.

\section{$3 \quad$ Experiments and results}

\section{Experimental protocol}

Experiments were conducted with a $5-10 \mathrm{MHz}$ linear array probe connected to a Dynamic Imaging Diasus ultrasound machine (http://www.dynamicimaging.co.uk). The depth setting was $4 \mathrm{~cm}$ with a single focus at $2 \mathrm{~cm}$. Analogue RF ultrasound signals were digitised after receive focusing and time-gain compensation, but before log-compression and envelope detection, using a Gage Compuscope CS14200 14-bit digitiser (http://www.gage-applied.com). Whole frames, each comprising $127 \mathrm{RF}$ vectors by 3818 samples, were stored in on-board Gage memory before transferring to $\mathrm{PC}$ memory at $75 \mathrm{MB} / \mathrm{s}$. The system operates in real time, with acquisition rates exceeding 30 frames per second. Sampling was at $66.67 \mathrm{MHz}$, synchronous with the ultrasound machine's internal clock: this synchronization minimises phase jitter between vectors. The acquired vectors were filtered with a 5-10 MHz filter, then envelope-detected using the Hilbert transform. The resulting $127 \times 3818$ frames of backscatter amplitude data formed the basis of all further computation. Their resolution is approximately $0.01 \mathrm{~mm}$ per sample in the axial direction and $0.3 \mathrm{~mm}$ per vector in the lateral direction.

The probe was mounted on a linear screw thread allowing accurate translation in the elevational and lateral directions. The probe's displacement was measured using a dial gauge. The scanning target was a speckle phantom [11] with Rayleigh backscatter and uniform attenuation of $0.4 \mathrm{~dB} / \mathrm{cm} / \mathrm{MHz}$. Each received B-scan was divided into an 8 column $\times 12$ row grid of patches. Lateral shifts were determined for each patch separately, by calculating the correlation with the preceding B-scan at candidate shifts, and using one of the aforementioned interpolation techniques to find $d_{l}^{*}$ to sub-vector precision. Axial shifts were assumed to be zero. The in-plane displacement of each B-scan relative to its predecessor was estimated using a three-parameter ( $x, y$ and roll) least squares fit to the grid of $d_{l}^{*}$ values. Similarly, elevational shifts $d_{e}$ were determined for each patch separately, using the calculated $\rho^{*}$ values, followed by a three-parameter ( $z$, tilt and yaw) least squares fit to the grid of $d_{e}$ values to find each B-scan's out-of-plane displacement. The elevational decorrelation curves, one for each patch, were obtained by scanning the same speckle phantom and measuring the decorrelation at different elevational offsets. Since we used the same phantom for both calibration and reconstruction, and since there is no probe rotation, tissue compression or physiological motion, we can be confident that reconstruction errors are due mainly to interpolation artefacts and not the other effects mentioned in Section 1.

The phantom was scanned in two patterns, as illustrated in Figure 4. In the first set of experiments, the probe was mounted parallel to the screw thread, resulting in pure lateral motion. $101 \mathrm{~B}$-scans were recorded at a pitch of $0.1 \mathrm{~mm}$. The accumulated lateral motion is therefore $10 \mathrm{~mm}$. In-plane reconstructions were attempted using every B-scan (stride 1), every other Bscan (stride 2), and so on up to stride 4 . Note that stride 3 corresponds to a single vector shift $(0.3 \mathrm{~mm})$ and therefore requires no interpolation: the stride 3 results will therefore allow us to 


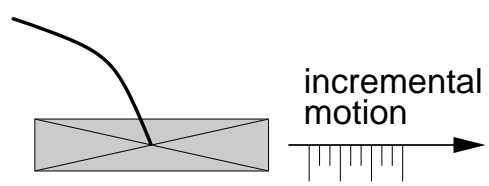

(a)

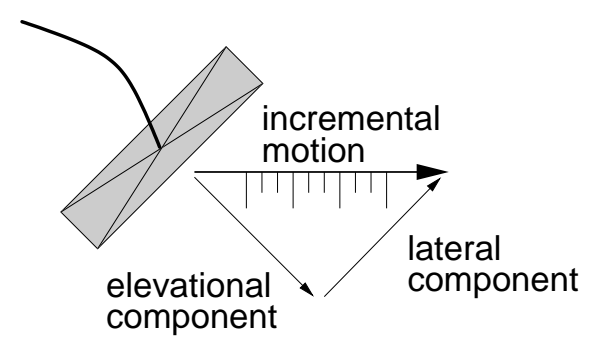

(b)

Figure 4: Experimental methodology. In the first set of experiments (a), the probe was moved step by step in the lateral direction, with B-scans recorded after each step. Each step was a third of the distance between A-lines, so three steps corresponds to a single A-line shift (as illustrated by the tick marks). In the second set of experiments (b), the probe was mounted at $45^{\circ}$ to the direction of motion, and the step size lengthened by approximately $\sqrt{2}$. Three steps still corresponds to a single A-line shift in the lateral direction, but there is now equal motion in the elevational direction.

assess how well the experimental design has eliminated other sources of reconstruction error. In the second set of experiments, the probe was mounted at $45^{\circ}$ to the screw thread. 101 B-scans were recorded, but this time at a pitch of $0.15 \mathrm{~mm}$. The total lateral and elevational motion is therefore $10.6 \mathrm{~mm}$ in each direction, and again little interpolation ${ }^{1}$ is required for a stride of 3 .

The experimental protocol can be summarised as follows. The steps marked $*$ are omitted in the first set of experiments, where there is no elevational motion.

1. Set $n \leftarrow 1$.

2. For each patch in B-scan $n$, estimate its lateral shift $d_{l}^{*}$ in B-scan $n+s$, where $s$ is the stride. Calculate the normalised correlation coefficient at a number of candidate shifts, then obtain $d_{l}^{*}$ to sub-vector precision using the techniques described in Section 2. For linear and Fourier interpolation, upsample the data by a factor of 15 .

3. * For each patch in B-scan $n$, use $d_{l}^{*}$ to find $\rho^{*}$, using either the peak of the fitted Gaussian or one further correlation with linear/Fourier data interpolation.

4. * Use $\rho^{*}$ and the calibrated elevational decorrelation curves to obtain the elevational offset $d_{e}$ for each patch.

5. Estimate the in-plane displacement ( $x, y$ and roll) between B-scans $n$ and $n+s$ using a least squares fit to the grid of $d_{l}^{*}$ values.

6. * Estimate the out-of-plane displacement ( $z$, tilt and yaw) between B-scans $n$ and $n+s$ using a least squares fit to the grid of $d_{e}$ values.

7. Let $n \leftarrow n+s$. While $n+s \leq 101$, repeat from step 2 .

8. Concatenate the displacements to obtain the total displacement between the final B-scan $N$ and B-scan 1. $N=101$ for strides of 1,2 and $4, N=100$ for a stride of 3.

The concatenated displacements offer an indirect but excellent way to assess the interpolation algorithms. Since each least squares fit involves the results of up to 96 interpolation calculations (one for each patch), any significant errors in the inter-frame displacements can be attributed to interpolation bias and not the random effects of a small number of calculations. Furthermore,

\footnotetext{
${ }^{1}$ Precisely no interpolation would have been required had the inter-frame displacement been $0.1 \times \sqrt{2} \mathrm{~mm}$ and not $0.15 \mathrm{~mm}$. The latter was chosen for convenience when reading the dial gauge.
} 


\begin{tabular}{l|lr|lr|lr|lrr} 
& \multicolumn{2}{|c|}{ stride 1 } & \multicolumn{2}{c|}{ stride 2 } & \multicolumn{2}{c|}{ stride 3 } & \multicolumn{2}{c}{ stride 4 } \\
& length & roll & length & roll & length & roll & length & roll \\
\hline correct & 10.00 & 0.00 & 10.00 & 0.00 & 9.90 & 0.00 & 10.00 & 0.00 \\
triangular & 6.51 & -0.08 & 11.61 & -0.35 & 9.82 & -0.17 & 9.12 & -0.21 \\
gaussian & 8.38 & 0.88 & 10.71 & -0.84 & 9.79 & -0.31 & 9.57 & 0.09 \\
offset gaussian & 9.99 & 1.04 & 9.89 & -0.90 & 9.80 & -0.31 & 9.94 & 0.07 \\
grid slopes & 9.85 & -0.70 & 9.94 & 0.00 & 9.69 & -0.42 & 9.97 & -0.29 \\
linear & 8.00 & 0.44 & 10.83 & -0.59 & 9.88 & -0.11 & 9.47 & -0.09 \\
fourier & 5.26 & -0.44 & 12.24 & -0.17 & 9.89 & -0.08 & 8.84 & -0.31
\end{tabular}

Table 1: Accumulated lateral length and roll for purely lateral probe motion. The lengths are in millimetres and the roll angles in degrees. The values were obtained by concatenating the in-plane transformations between neighbouring B-scans in the reconstruction sequence. There were 100 concatenations for a stride of 1,50 for a stride of 2,33 for a stride of 3 and 25 for a stride of 4 .

any dependency of the bias on the patch's location in the B-scan may lead to orientation errors in the inter-frame displacements, something we would not have noticed had we simply averaged the results across all the patches. Finally, the concatenated displacements are the natural quality measure for our particular application, sensorless freehand 3D ultrasound.

One final detail concerns the treatment of $\rho^{*}$ values greater than unity. These sometimes arise when fitting Gaussians and offset Gaussians, since there is no constraint that the peak be less than one. Such values were simply ignored in all subsequent analysis: so no $d_{e}$ estimate was obtained for the corresponding patch, and the patch did not contribute to the least squares estimation of the out-of-plane parameters. Patches were also ignored if they were found to contain excessive noise or if they contained artefacts from the phantom's scanning window (this affected the top two rows of patches): further details can be found in [8].

\section{Results}

The results for the first set of experiments, with purely lateral probe motion, are presented in Table 1. The table shows the accumulated lateral offset and roll found by concatenating the set of in-plane displacements for different strides. Roll arises predominantly as a result of a depthdependent interpolation bias. For example, a right bias in the top half of the B-scan coupled with a left bias in the bottom half would result in a gradual clockwise roll ${ }^{2}$. We have not tabulated the accumulated axial offsets since these were insignificant, as expected given the purely lateral probe motion.

Several conclusions can be drawn from Table 1. First, the uniformly good results for a stride of 3 confirm that the experimental design has successfully eliminated other sources of error: the less good results for other strides are due to interpolation. Second, all techniques exhibit significant bias for some strides, apart from the grid slopes and offset Gaussian approaches. The Fourier results suggest that the sampling frequency is not safely above the Nyquist limit, a hypothesis we confirmed by examining the spectra of several typical B-scan rows. Finally, we note a significant difference in performance between the two Gaussian techniques: it would appear that the offset is necessary to adequately capture the shape of the true decorrelation curve away from the focus.

The results for the second set of experiments, with both lateral and elevational probe motion, are presented in Tables $2-4$. Table 2 contains the accumulated in-plane offsets. The first thing to

\footnotetext{
${ }^{2}$ Roll could also arise through a left to right bias dependency, though we did not observe this to any degree. The behaviour of the interpolation techniques varied, to a greater or lesser extent, with vertical position in the B-scan, not horizontal position. This is not unexpected: the resolution cell varies significantly with depth, and this affects the spectrum of one-dimensional signals sampled from different rows of the B-scan. Depth-dependent interpolation bias may therefore be explained as an unsurprising sensitivity of the interpolation algorithm to frequency. There is no comparable effect operating left to right across the B-scans.
} 


\begin{tabular}{l|lr|lr|lr|lr} 
& \multicolumn{2}{|c|}{ stride 1 } & \multicolumn{2}{c|}{ stride 2 } & \multicolumn{2}{c|}{ stride 3 } & \multicolumn{2}{c}{ stride 4 } \\
& length & roll & length & roll & length & roll & length & roll \\
\hline correct & 10.61 & 0.00 & 10.61 & 0.00 & 10.50 & 0.00 & 10.61 & 0.00 \\
triangular & 7.05 & 0.23 & 12.20 & -0.55 & 10.11 & -0.18 & 9.76 & 0.19 \\
gaussian & 8.71 & 1.02 & 11.28 & -1.42 & 10.30 & 0.09 & 9.93 & 0.44 \\
offset gaussian & 10.33 & 1.40 & 10.46 & -1.68 & 10.33 & 0.02 & 10.10 & 0.70 \\
grid slopes & 10.84 & -0.79 & 9.74 & -0.28 & 10.27 & -0.34 & 10.31 & 1.58 \\
linear & 8.68 & 0.51 & 11.45 & -0.73 & 10.21 & -0.21 & 10.01 & 0.64 \\
fourier & 5.78 & -0.02 & 12.84 & -0.38 & 10.08 & -0.14 & 9.63 & -0.03
\end{tabular}

Table 2: Accumulated lateral length and roll for combined lateral and elevational probe motion. See the caption to Table 1 for a full description of the tabulated values.

\begin{tabular}{l|lr|lr|lr|lr} 
& \multicolumn{2}{|c|}{ stride 1 } & \multicolumn{2}{c|}{ stride 2 } & \multicolumn{2}{c|}{ stride 3 } & \multicolumn{2}{c}{ stride 4 } \\
& length & tilt & length & tilt & length & tilt & length & tilt \\
\hline correct & 10.61 & 0.00 & 10.61 & 0.00 & 10.50 & 0.00 & 10.61 & 0.00 \\
offset gaussian peak & 16.87 & 7.33 & 11.65 & 2.16 & 9.90 & 0.00 & 10.72 & 4.29 \\
linear & 21.71 & 15.39 & 13.49 & 5.68 & 10.49 & -0.05 & 11.49 & 3.31 \\
fourier & 23.61 & 20.29 & 14.23 & 7.95 & 10.66 & 0.28 & 11.76 & 4.15
\end{tabular}

Table 3: Accumulated elevational length and tilt for combined lateral and elevational probe motion. The lengths are in millimetres and the tilt angles in degrees. The values were obtained by concatenating the out-of-plane transformations between neighbouring B-scans in the reconstruction sequence. There were 100 concatenations for a stride of 1, 50 for a stride of 2, 33 for a stride of 3 and 25 for a stride of 4 .

note is the general degradation of the results for a stride of 3 , where little interpolation should be required. This is because the elevational motion causes some decorrelation between the patches, even when correctly aligned laterally. The correlation peak is no longer necessarily indicative of the correct lateral offset. Notwithstanding this, the grid slopes and offset Gaussian techniques still perform reasonably well. The marginal winner is the offset Gaussian technique, with a root mean square length error of $0.62 \mathrm{~mm}$ compared with $0.98 \mathrm{~mm}$ for grid slopes. A key assumption behind the grid slopes algorithm is that the auto- and cross-correlation functions of the two patches should be identical when there is no lateral motion: this assumption is violated when there is elevational motion.

Table 3 shows the accumulated elevational length and tilt. The linear and Fourier results were based on patch offsets $d_{l}^{*}$ found using the offset Gaussian technique. We have not tabulated the yaw angles, since these were small and unaffected by interpolation artefacts: as previously discussed, the interpolation bias may vary with depth (giving rise to tilt), but not left-right B-scan position.

The very poor results for a stride of 1 have little to do with interpolation, but are instead a consequence of the shape of the elevational decorrelation curve. As illustrated in Figure 1, the curve is fairly flat for small elevational separation, so the smallest error in $\rho^{*}$ gives rise to a far larger error in $d_{e}$. Attempting to reconstruct a data set with a stride of 1 is essentially an ill-conditioned problem [10]. This effect is certainly absent by the time we get to a stride of 3 , where linear and Fourier data interpolation give good values of $\rho^{*}$ and consequently good overall reconstructions. This is because little interpolation is actually required: the lateral correlation peak is at a shift of approximately one vector. For strides of 2 and 4, however, linear and Fourier data interpolation give unacceptably biased values of $\rho^{*}$ and hence poor reconstructions.

Considering strides in the range 2-4, the offset Gaussian peak would appear to be the most reliable way of estimating $\rho^{*}$. Even though there is evidence of significant bias for strides of 2 and 3 , it is important to realise that a real freehand data set is not going to exhibit uniform lateral shifts of, say, precisely one vector (stride 3 ) between B-scans. In practice, freehand reconstructions 


\begin{tabular}{l|ll|lr} 
& \multicolumn{2}{|c|}{ lateral } & \multicolumn{2}{c}{ elevational } \\
& length & roll & length & tilt \\
\hline correct & 10.61 & 0.00 & 10.61 & 0.00 \\
reconstruction 1 & 10.11 & 1.09 & 10.52 & 2.60 \\
reconstruction 2 & 10.25 & 0.20 & 10.59 & 2.17 \\
reconstruction 3 & 10.11 & 0.87 & 10.66 & 3.02
\end{tabular}

Table 4: Accumulated reconstruction lengths and angles for combined lateral and elevational probe motion. The lengths are in millimetres and the angles in degrees. The values were obtained by concatenating the in- and out-of-plane transformations between neighbouring B-scans in the reconstruction sequence. B-scans were selected at random strides in the range 2 to 4 . The offset Gaussian technique was used to obtain both $d_{l}^{*}$ and $\rho^{*}$ for all patches.

are going to require lateral interpolation at a range of shifts. Table 4 shows lengths and angles for three reconstructions of the second data set using the offset Gaussian peak and a random sequence of strides in the range 2 to 4 . Lateral displacement is correct to within $5 \%$, elevational displacement to within $1 \%$.

\section{Conclusions and further work}

Lateral interpolation has been largely ignored in the sensorless freehand 3D ultrasound literature. In this paper, we have shown that great care needs to be taken to minimise the effects of interpolation bias on reconstruction accuracy. Through a series of in vitro experiments, we have shown how fitting an offset Gaussian to the lateral correlation samples, and using the fitted Gaussian to determine both the location and magnitude of the correlation peak, gives the best results compared with other techniques. Nevertheless, interpolation bias can still lead to reconstruction errors of around $5 \%$ in length.

In future work, we will use offset Gaussian interpolation to reconstruct genuine freehand scans of in vivo tissue. It will be difficult, perhaps impossible, to decouple any interpolation artefacts from the other sources of error mentioned in Section 1 but, having established how to minimise each source of error as far as is possible, we are now in a better position to assess the achievable accuracy of sensorless freehand 3D ultrasound.

An important piece of work which needs to be done first, however, concerns frame selection. In a true freehand sequence, the inter-frame separation varies from frame to frame. Concatenating the displacements between all adjacent frames is unlikely to produce an optimal reconstruction, particularly if the frames are closely spaced and therefore on the flat part of the elevational decorrelation curve. So algorithms need to be developed to select interleaved subsets of frames for reconstruction. Subsequently, the separate, interleaved reconstructions must be combined into a final consensus result.

\section{Acknowledgments}

The authors would like to thank Joel Lindop for many helpful discussions.

\section{References}

[1] O. Bonnefous. Measurement of the complete (3D) velocity vector of blood flows. In Proceedings of the IEEE Ultrasonics Symposium, pages 795-799, 1988.

[2] R. Boucher and J. Hassab. Analysis of discrete implementation of generalized cross correlator. IEEE Transactions of Acoustics, Speech and Signal Processing, 29(3):609-611, 1981. 
[3] I. Cespedes, Y. Huang, J. Ophir, and S. Spratt. Methods for estimation of subsample time delays of digitized echo signals. Ultrasonic Imaging, 17(2):142-171, 1995.

[4] J-F. Chen, J. B. Fowlkes, P. L. Carson, and J. M. Rubin. Determination of scan-plane motion using speckle decorrelation: Theoretical considerations and initial test. International Journal of Imaging Systems Technology, 8:38-44, 1997.

[5] P. G. M. de Jong, T. Arts, A. P. Hoeks, and R. S. Reneman. Experimental evaluation of the correlation interpolation technique to measure regional tissue velocity. Ultrasonic Imaging, $13(2): 145-61,1991$.

[6] P. G. M. de Jong, T. Arts, A. P. G. Hoeks, and R. S. Reneman. Determination of tissue motion velocity by correlation interpolation of pulsed ultrasonic echo signals. Ultrasonic Imaging, 12(2):84-98, 1990.

[7] S. G. Foster, P. M. Embree, and W. D. O'Brien Jr. Flow velocity profile via time-domain correlation: error analysis and computer simulation. IEEE Transactions on Ultrasonics, Ferroelectrics and Frequency Control, 37(3):164-175, 1990.

[8] A. H. Gee, R. J. Housden, P. Hassenpflug, G. M. Treece, and R. W. Prager. Sensorless freehand 3D ultrasound in real tissue: speckle decorrelation without fully developed speckle. Medical Image Analysis, in press.

[9] B. J. Geiman, L. N. Bohs, M. E. Anderson, S. M. Breit, and G. E. Trahey. A novel interpolation strategy for estimating subsample speckle motion. Physics in Medicine and Biology, 45(6):1541-1552, 2000.

[10] P. Hassenpflug, R. Prager, G. Treece, and A. Gee. Distance measurement for sensorless 3D US. In Medical Image Computing and Computer-Assisted Intervention - MICCAI'04, pages 1087-1088, Saint-Malo, France, September 2004. LNCS 3217, Springer.

[11] J. M. Kofler and E. L. Madsen. Improved method for determining resolution zones in ultrasound phantoms with spherical simulated lesions. Ultrasound in Medicine and Biology, 27(12):1667-1676, 2001.

[12] M. Li. System and method for 3D medical imaging using 2D scan data. United States patent 5,582,173, application number 529778, September 1995.

[13] J. J. More. The Levenberg-Marquardt algorithm: implementation and theory. In A. Watson, editor, Numerical Analysis, pages 105-116. Lecture Notes in Mathematics 630, SpringerVerlag, 1977.

[14] R. W. Prager, A. H. Gee, G. M. Treece, C. J. C. Cash, and L. H. Berman. Sensorless freehand 3D ultrasound using regression of the echo intensity. Ultrasound in Medicine and Biology, 29(3):437-446, March 2003.

[15] G. M. Treece, R. W. Prager, A. H. Gee, and L. Berman. Correction of probe pressure artifacts in freehand 3D ultrasound. Medical Image Analysis, 6(3):199-215, 2002.

[16] T. A. Tuthill, J. F. Krücker, J. B. Fowlkes, and P. L. Carson. Automated three-dimensional US frame positioning computed from elevational speckle decorrelation. Radiology, 209(2):575$582,1998$.

[17] R.F. Wagner, M. F. Insana, and D. G. Brown. Statistical properties of radio-frequency and envelope-detected signals with applications to medical ultrasound. Journal of the Optical Society of America A, 4(5):910-922, May 1987.

[18] R.F. Wagner, M. F. Insana, and S. W. Smith. Fundamental correlation lengths of coherent speckle in medical ultrasonic images. IEEE Transactions on Ultrasonics, Ferroelectrics, and Frequency Control, 35(1):34-44, January 1988. 